\title{
LINAC OPTICS FOR ENERGY RECOVERY LINAC*\#
}

\author{
I. V. Bazarov, Cornell University, Ithaca, NY 14853, USA \\ G. A. Krafft, L. Merminga, TJNAF, Newport News, VA 23606, USA
}

\begin{abstract}
Several possible scenarios of Energy Recovery Linac (ERL) beam optics design are investigated to support the low emittance high current $\mathrm{CW}$ electron beam needed to drive a new ERL based X-ray Source. It is shown by numerical simulations that sufficiently high multipass beam break-up (BBU) threshold current can be achieved in a straightforward one-pass one-linac ERL scenario. A simple guideline for choosing optimal linac and recirculating transport line optics is suggested to realize best possible BBU threshold current.
\end{abstract}

\section{INTRODUCTION}

Multipass, multi-bunch transverse BBU has been long known to be a potential limiting factor in the operation of high current linac-based recirculating accelerators $[1,2]$. The effect is worse in superconducting $\mathrm{rf}$ (srf) cavities because of higher Q's of the high order modes (HOM). Besides, this instability is exacerbated in long rf structures as opposed to short ones. ERL envisioned at Cornell University [3] will constitute both of these complications, since it will have long $(400 \mathrm{~m})$ superconducting $\mathrm{rf}$ structure at the heart of the machine.

Experimental demonstration of $\mathrm{CW}$ beam with a current of some fraction of an Ampere in linac-based recirculating structures capable of providing energy in the range of several GeVs is yet to be seen. The result of calculations of BBU threshold current performed for JLAB IR FEL Upgrade [4] is indicative of seriousness of the problem under consideration. A reported BBU threshold current obtained by numerical simulations is 75 $\mathrm{mA}$ for $170 \mathrm{MeV}$ recirculating srf structure. The challenge of designing $5 \mathrm{GeV}$ several hundred meter long recirculating srf structure for ERL, which should be able to support $100 \mathrm{~mA}$ beam, becomes evident.

\section{OPTICS CHOICE STRATEGY}

Important insights into the problem can be gained by studying a simple case of one recirculation and only one HOM. In this case, analytical solution for BBU threshold current $I_{\text {th }}$ exists [5]:

$$
I_{\mathrm{th}}=-\frac{1}{e} \frac{2 \omega_{\lambda}}{(R / Q)_{\lambda} Q_{\lambda} k_{\lambda}^{2}\left(R_{12} / p_{i}\right) \sin \left(\omega_{\lambda} t_{r}\right)},
$$

here $e$ - electron charge; $(R / Q)_{\lambda}, Q_{\lambda}$ - geometric shunt impedance and quality factor of the mode respectively; $\omega_{\lambda}=k_{\lambda} c-$ the mode's frequency ( $c-$ speed of light); $t_{r}$ - recirculation time; $R_{12}$ - recirculation transfer matrix

\footnotetext{
*Supported by NSF Coop. Agrmts NSF PHY 9809799, DMR-9713424

${ }^{\text {\#} S u p p o r t e d ~ b y ~ U S ~ D O E ~ N o . ~ D E-A C 05-84 E R 40150 ~}$
}

element in TRANSPORT [6] notations (divergence to transverse position); $p_{i}$ - initial momentum of the beam.

There are several things one can suggest doing by looking at expression (1) to mitigate this instability. The most obvious answer is to increase the injection energy of the beam into the recirculating rf structure. However, to make ERL power efficient will require adopting as low injection energy as possible to minimize the amount of beam power that goes to the dump. Another important solution to lessen BBU instability consists of lowering Q's for the HOMs. HOM damping is of fundamental importance and will have to be incorporated into srf cavity design. Finally, a proper choice of transport line optics is essential to achieve high BBU threshold in ERL. The latter can be reduced in part to optimization problem of pass-to-pass beam-transport matrix elements $R_{12}$ and $R_{34}$ along the lengthy srf structure.

Analytical solution for the value of BBU threshold current exists only for very simple cases similar to the one presented earlier. To determine BBU threshold for an actual machine configuration it is necessary to employ computer simulation codes. Such a two-dimensional simulation code (TDBBU) has been developed at JLAB [7]. The problem of $R_{12}\left(R_{34}\right)$ optimization allows analytical solution only in a limited number of simple cases [1]. Therefore, computer optimization routines were used in this work to obtain the desired lattice parameters for ERL by adjusting the strength of quadrupoles in the srf structure. These two simulation tools have been extensively used to obtain optimal linac optics design, and to test suggested simple guidelines (discussed below) for choosing linac and recirculating transport line optics that should allow sufficiently high BBU threshold current in ERL.

3 BEAM DYNAMICS IN ERL

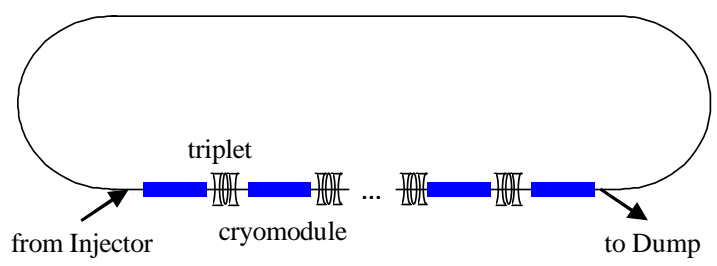

Fig. 1: Conceptual layout of ERL.

Conceptual layout of ERL is shown in Fig. 1. A 10 $\mathrm{MeV}$ electron beam with small normalized transverse emittance is introduced to the main linac. The srf structure accelerates the electrons to $5 \mathrm{GeV}$. Subsequently, the beam is taken through the recirculation transport loop where it is used to produce $\mathrm{x}$-rays. The beam is then 
returned to the linac with a $180^{\circ} \mathrm{rf}$ phase offset for energy recovery. In the linac the recirculated electrons give back their energy which is then being used for the acceleration of successive bunches. Finally, the low energy beam after energy recovery is taken to the dump.

Since srf structure extends to several hundred meters, it appears natural to introduce external quadrupole focusing in between cryomodules. External focusing in the linac is also critical to suppress BBU instability. For this purpose, quadrupole triplets in between srf cryomodules can be used because they allow more flexibility than doublets for example, acting more like an ideal thin lens. The fact that the bunches with two different energies are found along the linac introduces a major constraint on the focusing optics in the linac since electrons with different magnetic rigidity pass through the same quadrupoles. The constraint becomes especially important on the outside ends of the linac where energy ratio of the first pass to the second pass beams may be as high as 1000 (or about as high as 30 for the outer triplets inside the linac). This concern has led to the suggestion that the maximum energy ratio of two beams in the linac should not exceed $10[8,9]$. It would require splitting a $5 \mathrm{GeV}$ linac into two (or more) parts so that early stages of acceleration and late stages of energy recovery would take place in a section different from the longer srf structure where most of the acceleration / energy recovery occurs, e.g., see [9]. Nevertheless, we have found upon simulation that such a "split linac" scenario produced no advantages over a single long linac structure in terms of either BBU suppression or the ability to propagate a beam envelope with small $\beta$-functions through the linac.

\subsection{Adiabatic damping / antidamping}

Adiabatic damping / antidamping is another important reality to keep in mind when designing the linac optics. Consider the beam-transport matrix $(i \rightarrow f)$ for the drift with a uniform acceleration / deceleration:

$$
\left(\begin{array}{c}
x_{f} \\
x_{f}^{\prime}
\end{array}\right)=\left(\begin{array}{cc}
1 & \frac{\gamma_{i}}{\gamma} \ln \frac{\gamma_{f}}{\gamma_{i}} \\
0 & \frac{\gamma_{i}}{\gamma_{f}}
\end{array}\right)\left(\begin{array}{l}
x_{i} \\
x_{i}^{\prime}
\end{array}\right),
$$

here $\gamma$ and $\gamma^{\prime}$ are normalized energy (divided by electron rest energy $m c^{2}$ ) and accelerating gradient; $x$ is transverse position. $R_{12}=\frac{\gamma_{i}}{\gamma} \ln \frac{\gamma_{f}}{\gamma_{i}}$ can be interpreted as an effective length since $x_{f}-x_{i}^{\prime}=x_{i}^{\prime} R_{12}$ [10]. Thus, the effective length of the linac is contracted when the beam is accelerated ( $R_{12}=5 \mathrm{~m}$ with an actual $400 \mathrm{~m}$ linac) and elongated when the beam is decelerated $\left(R_{12}=2.5 \mathrm{~km}\right.$ if the beam is decelerated down to the energy of $10 \mathrm{MeV}$ ). This illustration indicates that special care must be taken in the late stages of the energy recovery where adiabatic antidamping is especially prominent.

\subsection{Rffocusing in standing-wave cavities}

In order to simulate correctly transverse dynamics of the particles in the linac, it is important to include rf focusing that an electron experiences when it traverses srf cavity. The study of rf focusing effect was carried out by different authors [11-13]. Following [13], the transport matrix for a pure $\pi$-mode standing-wave cavity for a particle run on rf-wave crest can be written as

$$
\left(\begin{array}{l}
x_{f} \\
x_{f}^{\prime}
\end{array}\right)=\left(\begin{array}{cc}
\cos (\alpha)-\sqrt{2} \sin (\alpha) & \sqrt{8} \frac{\gamma_{i}}{\gamma^{\prime}} \sin (\alpha) \\
-\frac{3}{\sqrt{8}} \frac{\gamma^{\prime}}{\gamma_{f}} \sin (\alpha) & \frac{\gamma_{i}}{\gamma_{f}}[\cos (\alpha)+\sqrt{2} \sin (\alpha)]
\end{array}\right)\left(\begin{array}{c}
x_{i} \\
x_{i}^{\prime}
\end{array}\right),
$$

here $\alpha=\frac{1}{\sqrt{8}} \ln \frac{\gamma_{f}}{\gamma_{i}}$. This expression also includes focusing (defocusing) kick that the particle experiences at the entrance (exit) of the cavity due to rf fringe field regions. This effect was implemented into TDBBU simulations.

\section{LINAC OPTICS SOLUTION}

As discussed earlier, the analytical solution (1) for the BBU threshold current in a simple case of one recirculation and one $\mathrm{HOM}$ suggests that BBU instability will be suppressed if the pass-to-pass matrix elements $R_{12}$ and $R_{34}$ can be made small everywhere in the srf cavities. Pass-to-pass matrix here is defined as the transport matrix from a given point in the linac to the same point after recirculation. As pointed out below, this statement represents a necessary, but not sufficient requirement for maximum BBU suppression in an actual recirculating accelerator. In general, the ideal condition $\left(R_{12}=R_{34}=0\right)$ cannot be satisfied everywhere in the linac except in the limit of no acceleration [1].

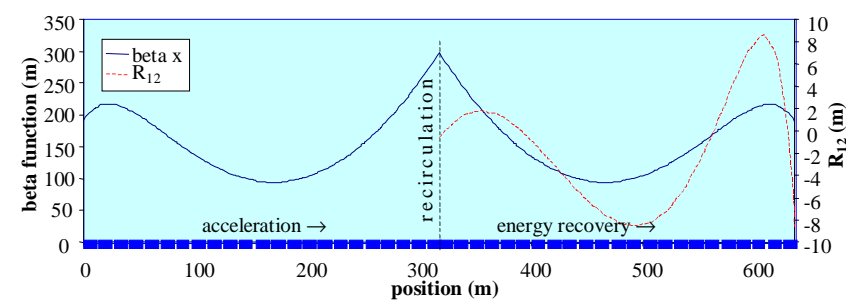

Fig. 2. $\beta$-function in the linac for the case of small $\mathrm{R}_{12}$ of pass-to-pass matrix (note a different scale for $\mathrm{R}_{12}$ plotted against the other ordinate axis on the right).

We have found that it is possible to produce an ERL configuration which will have small values of $R_{12}$ and $R_{34}(\leq 9 \mathrm{~m})$ for a long srf structure of several hundred meters (see also [8]). It is interesting to note that in this case absolutely no external focusing is required in the linac to achieve small values of $R_{12}$ and $R_{34}$. This corresponds to large $\beta$-functions in the linac (the maximum value is approximately equal to the total length of the srf structure). An example of the $\beta$-function in such a configuration, as well as $R_{12}$ values along the linac, is shown in Fig. 2 (the vertical $\beta_{y}$ - and horizontal $\beta_{x}$ functions, and $R_{12}$ and $R_{34}$ are chosen to match since the rf focusing in the linac is radially symmetric). The injection energy is $410 \mathrm{MeV}$ and the full energy is 5.01 $\mathrm{GeV}$. The srf structure of about $300 \mathrm{~m}$ consisted of 23 cryomodules, each providing $200 \mathrm{MeV}$ of energy. To obtain small values of $R_{12} \quad\left(R_{34}\right)$, the pass-to-pass betatron phase advance, $\Delta \psi$, has to be an integer multiple of $\pi$ throughout the linac. This can be achieved by adjusting optics in the turnaround arc.

Despite the fact that the obtained $R_{12}\left(R_{34}\right)$ values are 
small throughout the srf structure, TDBBU simulations for calculated $\mathrm{HOM}$ data taken from 9-cell $1.3 \mathrm{GHz}$ TESLA cavities [14] yielded an instability threshold current of less than $25 \mathrm{~mA}$. Such a low BBU threshold current can be explained by the fact that small values of $R_{12} \quad\left(R_{34}\right)$ for the pass-to-pass matrix do not guarantee suppression of the instability since the same matrix elements may be quite large for a transport matrix from cavity to cavity. In other words, although the condition discussed above does suppress the coupling of a HOM in the same cavity on successive passes, it does not preclude the HOMs of two cavities in different parts of the srf structure to "talk" to each other, either on the same or on successive passes, thus rendering the system unstable. Indeed, one can write the following expression for $R_{12}$ in terms of the $\beta$-function and phase advance

$$
R_{12}(i \rightarrow f)=\gamma_{i} \sqrt{\frac{\beta_{i}}{\gamma_{i}} \frac{\beta_{f}}{\gamma_{f}}} \sin \Delta \psi .
$$

According to (2), the $R_{12}\left(R_{34}\right)$ of the transport matrix between different cavities can be very large (on order of the $\beta$-function or even bigger). For the example under consideration, the phase advance in the srf structure changes rather slowly and consequently, the absolute value of $R_{12}$ between the first cryomodule on acceleration and other cryomodules along the linac during energy recovery increases slowly, reaching about $150 \mathrm{~m}$ in the last cryomodule.

These observations suggest the following guidelines for choosing focusing optics in the linac:

- $\quad$ Minimize the $\beta$-function in the linac by adjusting the strength of the quadrupoles and by matching the envelope of the injected beam.

- Set the phase advance of the recirculating arc to minimize $R_{12}$ and $R_{34}$ of the pass-to-pass matrix.
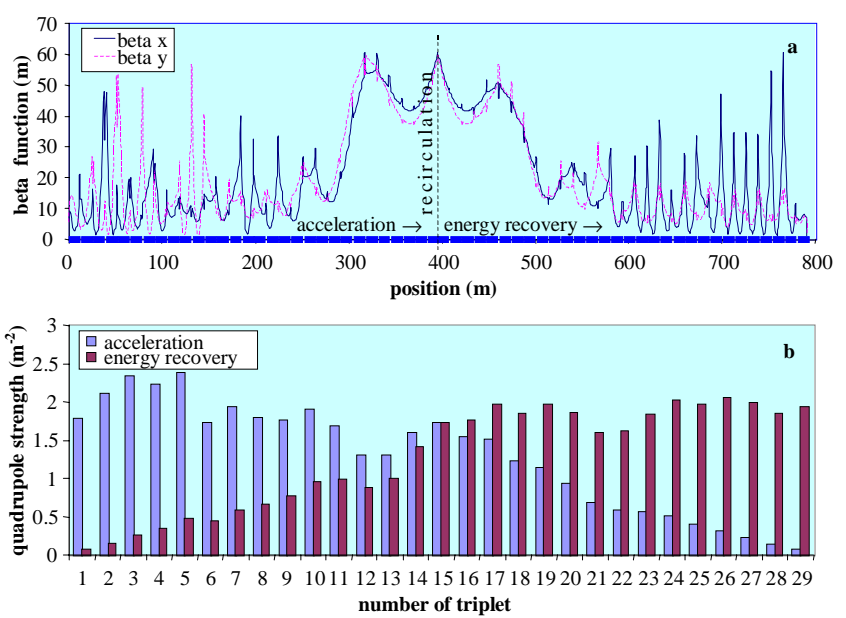

Fig. 3. Optimized linac optics: a) $\beta$-functions in the linac; b) corresponding strengths of quadrupoles.

Because of all the earlier mentioned complexities of transverse particle dynamics in the ERL, the first task of minimizing the $\beta$-function in the linac is best achieved by numeric optimization methods (for example, by employing a quasi-Newton method [15]).
The result of such optimization for a $5 \mathrm{GeV}$ ERL with accelerating gradient in cavities of $20 \mathrm{MV} / \mathrm{m}$ is presented in Fig. 3. The injection energy is $10 \mathrm{MeV}$. The $5 \mathrm{GeV}$ srf structure contains 30 cryomodules, and its length is about $400 \mathrm{~m}$. The strength, $|k|$ (where $k=(\partial B / \partial x) /(B \rho), B$ and $(B \rho)$ are magnetic field and rigidity respectively), of a middle quadrupole of all 29 triplets $(-1 / 2 k, k,-1 / 2 k$; quadrupoles are of the same 50-cm-length) is shown in Fig. 3b. It can be seen from Fig. 3b that triplets are set in such a way as to produce a nearly constant focusing length in the quadrupoles for the beam during the final stage of energy recovery. Simulations indicate BBU threshold current of $205 \mathrm{~mA}$ for HOM data of Table 1.

Table 1. HOM parameters used in BBU simulations [14].

\begin{tabular}{|c|c|c|c|}
\hline $\boldsymbol{f}$ (MHz) & polarization & $\boldsymbol{R} / \boldsymbol{Q}[\boldsymbol{\Omega}]$ & $\boldsymbol{Q}$ \\
\hline 1734 & $\mathrm{x} / \mathrm{y}$ & 116.7 & $3400 / 4500$ \\
\hline 1865 & $\mathrm{x} / \mathrm{y}$ & 42.4 & $50600 / 26500$ \\
\hline 1874 & $\mathrm{x} / \mathrm{y}$ & 56.8 & $50200 / 51100$ \\
\hline 1880 & $\mathrm{x} / \mathrm{y}$ & 11.8 & $95100 / 85500$ \\
\hline 1887 & $\mathrm{x} / \mathrm{y}$ & 1.2 & $633000 / 251000$ \\
\hline
\end{tabular}

\section{CONCLUSION}

Simple guidelines for choosing optimal linac optics for ERLs are suggested to realize best possible BBU threshold current.

\section{ACKNOWLEDGEMENTS}

Authors wish to thank D. Douglas and R. Talman for their help in this work.

\section{REFERENCES}

[1] R. E. Rand, T. I. Smith, Particle Accelerators 11, p. 113 (1980)

[2] J. J. Bisognano, R. L. Gruckstern, IEEE Proc. 1987

Part. Accel. Conf., p. 1078-1080 (1987)

[3] M. Tigner et al., these proceedings (2001)

[4] L. Merminga et al., IEEE Proc. 1999 Part. Accel. Conf., p. 1180-1182 (1999)

[5] G. A. Krafft et al., JLAB-TN-01-011, 1988

[6] K. L. Brown et al., SLAC-91, February 1974

[7] G. A. Krafft, J. J. Bisognano, IEEE Proc. 1987 Part. Accel. Conf., p. 1356-1358 (1987)

[8] D. Douglas, JLAB-TN-00-005, February 2000

[9] I. Ben-Zvi et al., Proc. $2^{\text {nd }}$ eRHIC Workshop, Yale University, April 2000

[10] R. Helm, R. Miller, in Linear Accelerators, edited by P. M. Lapostolle and A. L. Septire (North-Holland, Amsterdam, 1969)

[11] E. E. Chambers, HEPL TN-68-17, Stanford, (1968)

[12] G. A. Krafft, CEBAF TN-91-80, April 1991

[13] J. Rosenzweig, L. Serafini, Phys. Rev. E 49, p. 15991602 (1994)

[14] J. Sekutowicz, TESLA 94-07, February 1994

[15] P. E. Gill, W. Murray, Journal of the Institute of Mathematics and Its Applications 9, p. 91-108 (1972) 